\title{
Challenges in the modification of the M1 stage of the TNM staging system for nasopharyngeal carcinoma: A study of 1027 cases and review of the literature
}

\author{
CHANG-CHUAN PAN ${ }^{1,2}$, JIN LU $^{1}$, JING-RUI YU ${ }^{1}$, PING CHEN $^{1}$, WANG LI $^{2}$, ZI-LIN HUANG ${ }^{2}$, \\ MING ZHAO ${ }^{2}$, ZHI-MEI HUANG ${ }^{2}$, YUN-FEI XIA ${ }^{3}$, YAN-HEN WU ${ }^{4}$ and PEI-HONG WU ${ }^{2}$ \\ ${ }^{1}$ Medical Oncology, Sichuan Cancer Hospital and Institute, The Second People's Hospital of Sichuan Province, Sichuan; \\ Departments of ${ }^{2}$ Medical Imaging and Interventional Radiology, ${ }^{3}$ Radiation Oncology, and \\ ${ }^{4}$ State Key Laboratory of Oncology in South China, Sun Yat-Sen University Cancer Center, Guangzhou, P.R. China
}

Received February 24, 2012; Accepted May 3, 2012

DOI: $10.3892 /$ etm.2012.584

\begin{abstract}
A series of modifications have been introduced to the TNM staging system over time for nasopharyngeal carcinoma (NPC), mainly focused on the T (primary tumor) and $\mathrm{N}$ (local node) components of the system. The M1 stage is a 'catch all' classification, covering a group of patients whose outlook ranges from potentially curable to incurable. Since the current M1 stage does not allow clinicians to stratify patients according to prognosis or guide therapeutic decision-making and allow comparison of results of radical and non-radical treatments, we aimed to subdivide the M1 stage according to a retrospective study of 1027 metastatic NPC patients and to review the relevant literature. Between 1995 and 2007, 1027 inpatients with distant metastasis from NPC were retrospectively analyzed. Various possible subdivisions of the M1 stage were considered, looking at different metastatic sites, the number of metastatic organs and the number of metastases. Survival rates were calculated using the Kaplan-Meier method and compared using the log-rank test. The most frequently involved metastatic sites were the bone, lung and liver. The incidence rates of solitary metastatic lesions and pulmonary metastasis were 16.2 and $41.3 \%$. Despite the poor survival of these patients with a median survival of 30.8 months, patients in the metachronous metastatic group with metastases to the lung and/or solitary lesions, were defined as M1a, and were significantly associated with favorable median survival of 41.5 and 49.1 months in the univariate and multivariate analysis, respectively. Patients in the metachronous metastatic group
\end{abstract}

Correspondence to: Professor Pei-Hong Wu and Dr Ming Zhao, Department of Medical Imaging and Interventional Radiology, State Key Laboratory of Oncology in South China, Sun Yat-sen University Cancer Center, 651 Dongfeng Road East, Guangzhou 510060, P.R. China

E-mail: wuph@sysucc.org.cn and zhaoming@sysucc.org.cn

Key words: distant metastasis, M1 stage, nasopharyngeal carcinoma, metastatic survival, prognostic factors with metastasis to the lung and/or solitary lesions (M1a) have a more favorable prognosis compared with those patients with multiple metastases located in other anatomic sites (M1b). These data, in one of the largest reported metastatic NPC cohorts, are the first to show the prognostic impact of metastatic status in NPC. As a powerful predictor, the potential clinical value of a modified M1 of the TNM system for NPC will facilitate patient counseling and individualize management.

\section{Introduction}

The objectives of the TNM system are to aid clinicians and investigators in planning treatment, assessing prognosis, stratifying patients for therapeutic studies, evaluating the results of treatment and facilitating communication $(1,2)$. A series of modifications was introduced to the TNM staging system for nasopharyngeal carcinoma (NPC), while focusing on the primary tumor $(\mathrm{T})$ and local node $(\mathrm{N})$ components. However, the metastasis (M1) stage is a 'catch-all' classification, covering a group of patients whose outlook ranges from potentially curable to incurable, and does not allow clinicians to stratify patients according to prognosis or guide therapeutic decision making or allow comparison of results of radical and non-radical treatments.

In more recent years, increasing interest in the use of local therapy for metastases has arisen to prolong the life and improve the quality of life of patients with metastases including NPC and other solid tumors (3-8). Based on the fact that local therapy offers the chance of cure for patients with liver metastases in colorectal cancer, the European Colorectal Metastases Treatment Group in 2006 proposed a new M1 staging system that would subdivide the M1 into three subcategories (9). This proposal was accepted in seven editions of the AJCC cancer staging manual. The current challenge is to obtain a similar result in modifying the M1 stage of the TNM staging system for NPC. Studies have shown that liver metastasis is prognostic for metastatic survival in metastatic NPC and is associated with a shorter survival when compared with lung or bone metastases (4-6,10-12). However, there is a lack 
of large, systematic cohort studies on the prognostic impact of metastatic status in NPC. We aimed to subdivide the M1 stage according to our retrospective study of 1027 NPC patients with distant metastasis and a review of the literature.

\section{Patients and methods}

Patients. This retrospective study was approved by the Institutional Review Board of Sun Yat-Sen University Cancer Center. The institutional database was reviewed to identify 1027 inpatients with a confirmed clinical diagnosis of metastatic NPC between January 1995 and December 2007. The diagnosis of metastasis was based on histological evaluation, and/or combined with imaging and subsequent clinical followup confirmation. We excluded the 11 patients who did not have a complete medical history of clinical and follow-up data. We reviewed the clinical records of the remaining 1016 cases. Patient demographics, characteristics of their metastases and treatment modality were recorded using Epidate 3.0 software.

Study protocol. Subdivision of the M1 stage was attempted according to various metastatic sites, the number of metastatic organs and the number of metastases. The following prognostic variables were also assessed: synchronous versus metachronous onset of metastases, age ( $\leq 47$ or $>47$ years), gender, AJCC 1998 stage classification for $\mathrm{T}$ and $\mathrm{N}$ classification and body mass index (BMI) $(<18.5,18.5-24.9,>25)$. Patients were grouped according to the above factors, and survival analysis was carried out by subgroup. Metastatic survival was defined as survival from the first diagnosis of metastases to the time of death, or the cut-off day of the study using the Kaplan-Meier method. The survival status was verified on 31 August, 2009 through direct telecommunication with the patient or family and checking of the clinic attendance records. The log-rank test was used to assess the significance between variables. Analyses were performed using the SPSS 15.0 software package.

\section{Results}

Characteristics of metastases. This analysis included 1016 patients. Patient ages ranged from 15 to 78 years, with a median age of 45.8 years. At the time of the first diagnosis of metastases, 376 patients had solitary organ metastasis $(33.96 \%)$ and 164 patients had solitary metastatic lesions (16.15\%). The most frequently involved sites were bone (542, 53.35\%), lung $(420,41.34 \%)$ and liver $(302,29.72 \%)$. Following diagnosis of metastases, all were candidates for cytotoxic chemotherapy.

Metastatic overall survival and prognostic factors. The median overall survival was 30.8 months from the time of diagnosis of metastases for all of the 1016 NPC patients. For the 376 patients in the synchronous metastatic group, the median survival was 23.3 months and the 1-, 3- and 5-year metastatic overall survival rates were $74.2,27.6$ and $18.5 \%$, respectively. For the 640 patients in the metachronous metastatic group, the median survival was 36.7 months and the 1-, 3- and 5-year metastatic overall survival rates were 88.1, 49.6 and $28.6 \%$, respectively $(\mathrm{P}<0.001)$. Subdivisions of the M1

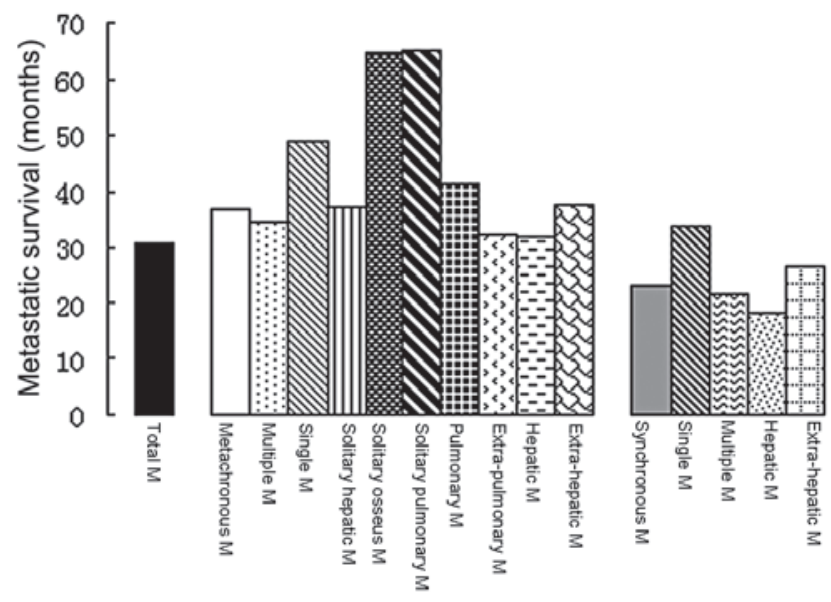

Subdividing the M1 stage by metastatic status

Figure 1. Metastatic survival in 1016 patients with metastatic nasopharyngeal carcinoma according to subdivisions of metastatic status.

stage according to other metastatic statuses were significantly associated with survival (Figs. 1 and 2).

In the univariate analysis among the metachronous and synchronous metastatic groups, various metastatic sites (liver, lung and bone) and the number of metastases (solitary) were significant prognostic factors. The number of metastatic organs was significantly associated with survival in the metachronous metastatic group (Fig. 3), but not in the synchronous metastatic group.

Upon multivariable analysis, in the metachronous metastatic group, various metastatic sites (pulmonary), the number of metastatic lesions (solitary) and $\mathrm{N}$ stage were identified as independent prognostic factors, while metastatic status was not associated with survival in the multivariate analysis (Table I).

\section{Discussion}

A number of published studies $(4-6,8,11,12)$, as reported in Table II, have focused on the characteristics of metastases, with the goal of identifying a relatively favorable prognostic group. However, these studies were limited by their small cohorts. The present study is the first to reflect the clinical course of metastatic NPC in a large cohort.

Given that patients with metastatic NPC may undergo substantially different clinical courses, we sought to improve the prediction of the prognosis of metastatic NPC by subdividing the M1 stage according to metastatic status, in order to categorize patients with metastatic NPC into groups with large differences in survival. Our results indicate that a subset of metastatic NPC patients with pulmonary metastasis and/or solitary lesions was potentially associated with a relatively favorable prognosis. In addition, in the present study, the $16.15 \%$ incidence of solitary lesions and the $41.34 \%$ incidence of pulmonary metastasis were high rates and should not be ignored.

One of the aims of a new staging system would be to allow the stratification of patients from the onset in terms of their potential curability and use this to direct their therapeutic management. In previously published results regarding hepatic 
A

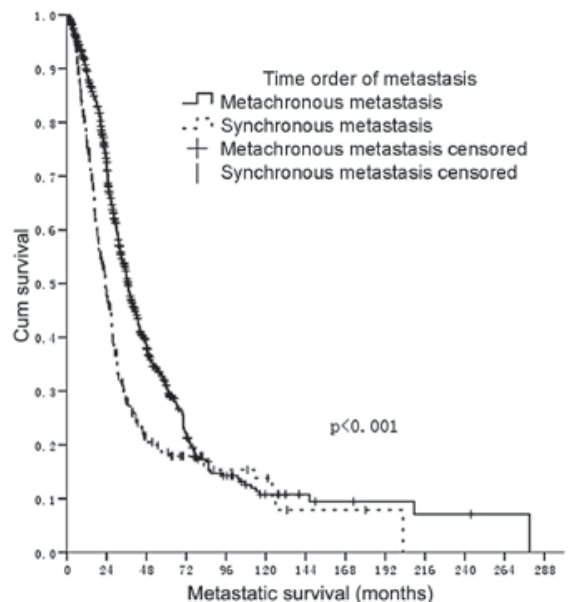

C

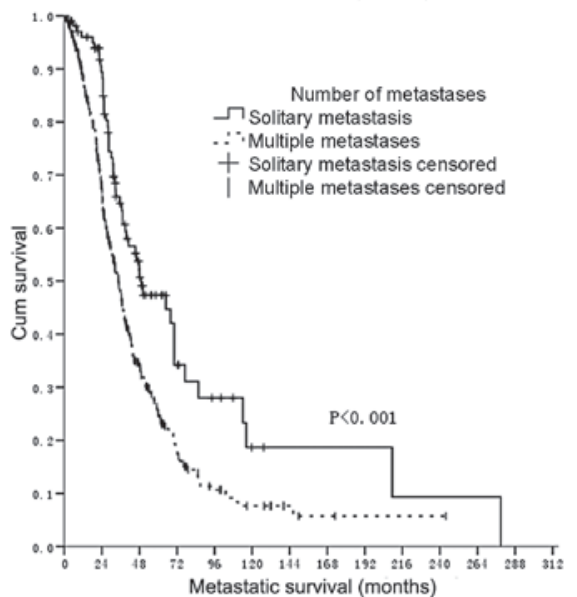

B

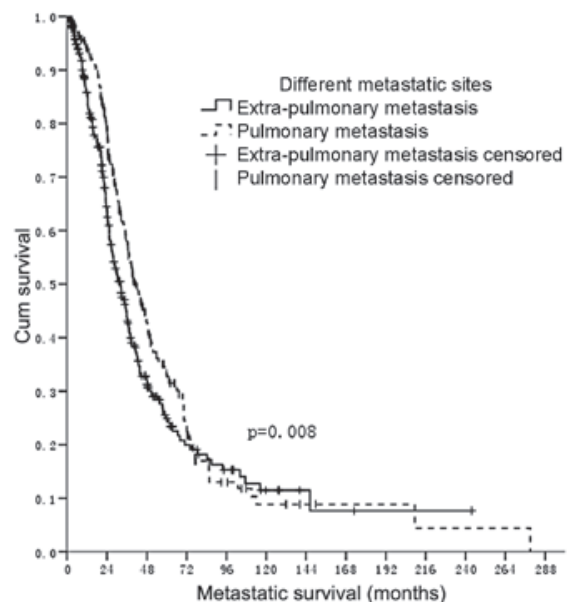

D

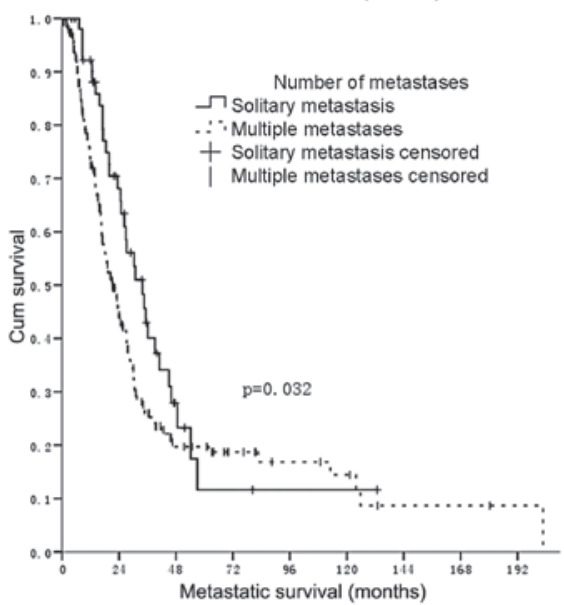

Figure 2. Metastatic survival in 1016 patients with metastatic nasopharyngeal carcinoma. (A) Survival grouped by time according to the diagnosis of metastasis. (B) Survival grouped by the presence or absence of pulmonary metastasis for 640 patients with metachronous metastasis. (C) Survival grouped by the number of metastases in 640 patients with metachronous metastasis. (D) Survival grouped by the number of metastases in 376 patients with synchronous metastasis.

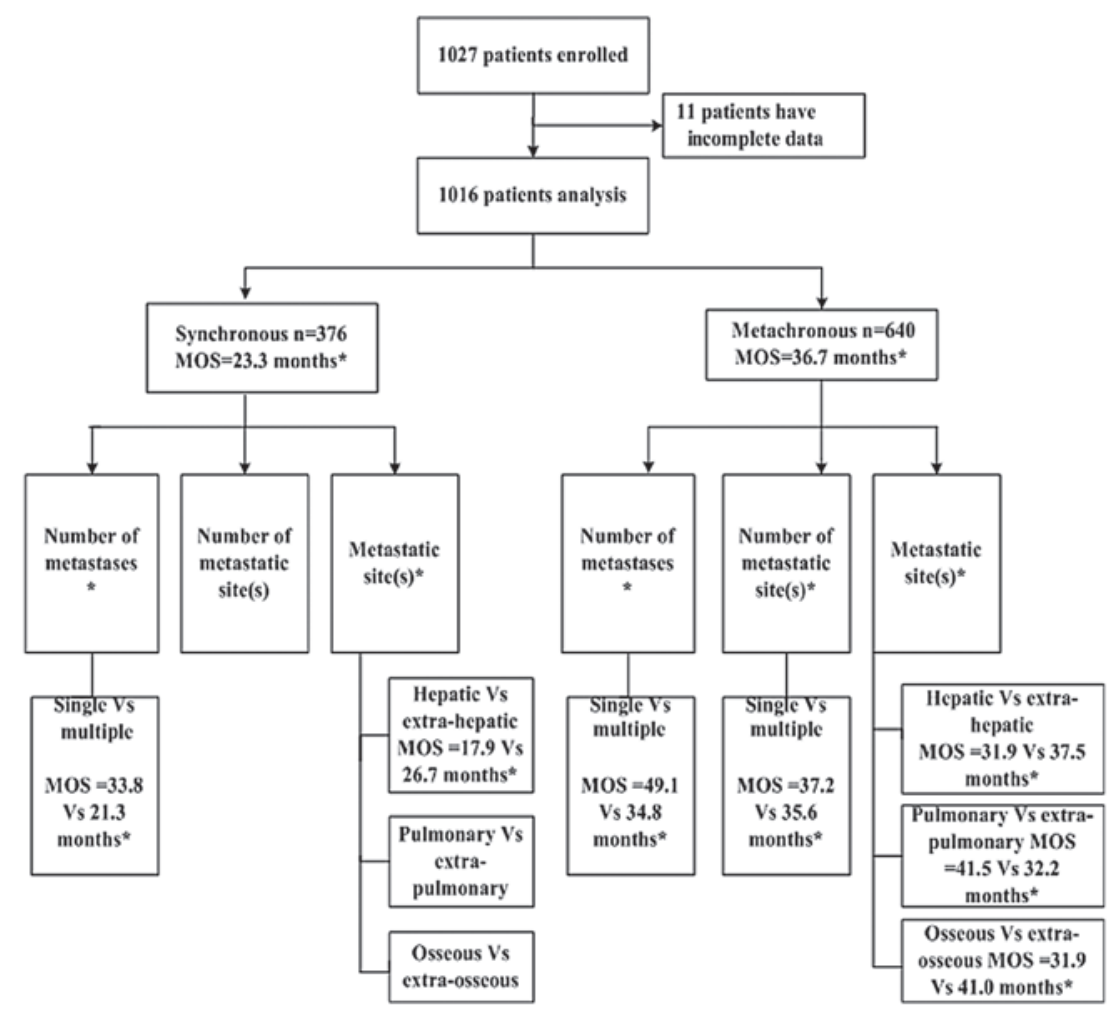

Figure 3. Study profile of the 1027 patients with metastatic nasopharyngeal carcinoma. ${ }^{*} \mathrm{p}<0.05$. 
Table I. Significantly independent variables from the multivariate analysis of 1016 patients with metastatic nasopharyngeal carcinoma.

\begin{tabular}{lll}
\hline Factors & HR (95\% CI) & P-value \\
\hline Metachronous metastatic group (n=640) & & \\
Number of metastases (solitary) & $0.37(0.21-0.52)$ & 0.003 \\
Metastatic sites (pulmonary) & $0.62(0.37-0.89)$ & 0.018 \\
N stage (1998) (N3) & $1.24(1.10-1.40)$ & 0.000 \\
Synchronous metastatic group (n=376) & $1.26(1.08-1.49)$ & 0.004 \\
N stage (1998) (N3) & $1.30(1.11-1.53)$ & 0.001 \\
BMI $(<18.5)$ & & \\
\hline
\end{tabular}

Table II. Survival of metastatic nasopharyngeal carcinoma patients in studies focusing on different metastatic status.

\begin{tabular}{|c|c|c|}
\hline First author (ref.) & No. of patients & Survival \\
\hline Pan et al $(4,6)$ & 376 & Median survival was 16.5 months for liver metastases \\
\hline Pan et al (5) & 480 & Median survival was 36.1 months for lung metastases \\
\hline Ma et al (8) & 105 & Median survival was 47.7 months for solitary lung metastases \\
\hline Hui et al (11) & 379 & $\begin{array}{l}\text { Median survival after primary radiotherapy was } 2.1 \text { vs } 3.9 \text { years } \\
\text { for the distant metastasis group vs. only lung metastasis group }\end{array}$ \\
\hline Khanfir et al (12) & 95 & $\begin{array}{l}\text { One-year metastatic survival rates were } 40 \% \text { (for all) and } 51 \% \text { vs. } 25 \% \\
\text { for the single metastasis group vs. the multiple metastasis group }\end{array}$ \\
\hline
\end{tabular}

and pulmonary metastases from NPC, selected groups of patients including patients with solitary metastasis benefit from local therapy. Some are potentially curable $(4-6,8)$. From the experience of subdividing the M1 stage of colorectal cancer, we hold that the development of such a staging system is of importance. A challenge facing the TNM staging system for NPC is how to modify the M1 stage for more precise prognostic prediction, thereby enabling a more tailored therapeutic approach with improved outcomes. The development of new staging strategies must be relevant to current clinical practice, be evidence-based and reflect the dominant prognostic factors consistently identified in Cox multivariate regression analyses. Based on our results and those published in the medical literature as reported in Table II, we propose that a status of metachronous metastatic NPC is suggested for classifying patients according to two categories. Patients with pulmonary metastasis and/or solitary lesions are categorized as M1a; they have a better prognosis compared with those patients with multiple metastases located in any other anatomic site (designated as M1b). The advantage of this suggested staging system is that it is simple and can be used for the daily care of patients with metastatic NPC. Although it is likely that a more detailed and perhaps more sophisticated M1 staging system may be found to stratify patients entered in clinical trials, these pilot results and modifications may be currently useful in the design of clinical trials for metastatic NPC, for it is able to more accurately stratify patients into groups with fairly consistent outcome and thus help to standardize the reported results of any therapeutic interventions, with fewer samples and less financial expenditure.
Our results and modifications should be interpreted in the light of some limitations, including the quality of retrospective data from a single clinical center and the small number of cases. Therefore, our results should be further validated by additional studies to ensure its potential clinical value.

In conclusion, this study represents a single institutional experience of distinguishing the prognostic significance of different metastatic statuses of NPC and subdividing the M1 stage of metastatic NPC. Our findings have the potential to provide clinicians with useful, easily available information for personalizing therapy.

\section{References}

1. Greene FL and Sobin LH: The staging of cancer: a retrospective and prospective appraisal. CA Cancer J Clin 58: 180-190, 2008.

2. Gospodarowicz MK, Miller D, Groome PA, Greene FL, Logan PA and Sobin LH: The process for continuous improvement of the TNM classification. Cancer 100: 1-5, 2004.

3. Timmerman RD, Bizekis CS, Pass HI, et al: Local surgical, ablative, and radiation treatment of metastases. CA Cancer J Clin 59: 145-170, 2009.

4. Pan C, He N, Zhao M, et al: Subdividing the M1 stage of liver metastasis for nasopharyngeal carcinoma to better predict metastatic survival. Med Oncol 28: 1349-1355, 2011.

5. Pan CC, Wu PH, Yu JR, et al: Comparative survival analysis in patients with pulmonary metastases from nasopharyngeal carcinoma treated with radiofrequency ablation. Eur J Radiol 81: 473-477, 2012.

6. Pan C, Wu P, Yu J, et al: CT-guided radiofrequency ablation prolonged metastatic survival in patients with liver metastases from nasopharyngeal carcinoma. Int J Hyperthermia 27: 549-554, 2011. 
7. Tomlinson JS, Jarnagin WR, DeMatteo RP, et al: Actual 10-year survival after resection of colorectal liver metastases defines cure. J Clin Oncol 25: 4575-4580, 2007.

8. Ma J WZ, Lin P, Wang X, Xie FY, Ma J, Wen ZS, Lin P, Wang X and Xie FY: The results and prognosis of different treatment modalities for solitary metastatic lung tumor from nasopharyngeal carcinoma: a retrospective study of 105 cases. Chin J Cancer 29: 787-795, 2010.

9. Nordlinger B, Van Cutsem E, Rougier P, et al: Does chemotherapy prior to liver resection increase the potential for cure in patients with metastatic colorectal cancer? A report from the European Colorectal Metastases Treatment Group. Eur J Cancer 43: 2037-2045, 2007.
10. Ong YK, Heng DM, Chung B, et al: Design of a prognostic index score for metastatic nasopharyngeal carcinoma. Eur J Cancer 39: 1535-1541, 2003

11. Hui EP, Leung SF, Au JSK, et al: Lung metastasis alone in nasopharyngeal carcinoma: a relatively favorable prognostic group - a study by the Hong Kong nasopharyngeal carcinoma study group. Cancer 101: 300-306, 2004.

12. Khanfir A, Frikha M, Ghorbel A, Drira MM and Daoud J: Prognostic factors in metastatic nasopharyngeal carcinoma. Cancer Radiother 11: 461-464, 2007. 